\title{
COMPACT MANIFOLDS \\ WITH A LITTLE NEGATIVE CURVATURE
}

\author{
K. D. ELWORTHY AND S. ROSENBERG
}

1. Bochner's Theorem states that a compact oriented Riemannian manifold $(M, g)$ with positive Ricci curvature has $H^{1}(M ; \mathbf{R})=0$. Myers' Theorem implies the stronger result that $\pi_{1}(M)$ is finite under the same hypothesis. Both theorems fail if the Ricci curvature is positive except on a set of arbitrarily small diameter, since every compact manifold admits such a metric of volume one. Nevertheless, we can extend these theorems and the Bochner Theorem for $p$-forms, yielding topological obstructions to manifolds admitting metrics with a little negative curvature.

2. Results for $H^{1}(M ; \mathbf{R})$. The Laplacian on $p$-forms has the Weitzenböck decomposition $\Delta^{p}=\nabla^{*} \nabla+R^{p}$; here $\nabla$ is the Levi-Civita connection and $R^{p} \in \operatorname{End}\left(\Lambda^{p} T^{*} M\right)$ with $R^{1}=$ Ricci. We write $R^{p}(x) \geq R_{0}$ for $x \in M$ if the lowest eigenvalue of $R^{p}(x)$ is at least $R_{0}$. In what follows, we normalize all metrics to have volume one.

Theorem 1. Pick $R_{0}>0$ and $K<0$. There exists $\varepsilon=\varepsilon\left(R_{0}, K, \operatorname{dim} M\right)$ $>0$ such that if $\operatorname{Ric}(x) \geq R_{0}$ except on a set $A$, with diameter $\operatorname{diam}(A) \leq \varepsilon$, where $\operatorname{Ric}(x) \geq K$, then $H^{1}(M ; \mathbf{R})=0$.

In other words, if the metric has a deep well of negative Ricci curvature, we may still conclude $H^{1}(M ; \mathbf{R})=0$ provided the well is narrow enough. Notice that there is no restriction on the topology of $A$.

Theorem 1 is a consequence of the following weaker version about metrics with a shallow well of negative Ricci curvature.

Theorem 1'. Pick $R_{0}>0$. There exists $\varepsilon^{\prime}=\varepsilon^{\prime}\left(R_{0}, \operatorname{dim} M\right)>0$ and $\delta=\delta\left(R_{0}, \operatorname{dim} M\right)<0$ such that if $\operatorname{Ric}(x) \geq R_{0}$ except on a set $A$, with $\operatorname{diam}(A) \leq \varepsilon^{\prime}$, where $\operatorname{Ric}(x) \geq \delta$, then $H^{1}(M ; \mathbf{R})=0$.

We sketch a proof of Theorem $1^{\prime}$. By semigroup domination for the heat flow on one forms, it is enough to show that $\Delta^{0}+\mathrm{Ric}^{\prime}>0$, where $\operatorname{Ric}^{\prime}(x)$ is the lowest eigenvalue of Ricci at $x$. By an elementary argument, we have

LEMMA 2. Let $V: M \rightarrow \mathbf{R}$ be continuous. If (i) $\int_{M} V d \operatorname{vol}(g)>0$ and

(ii) $\lambda_{1} \geq-V_{\min }+\frac{\left\|V-V_{a v}\right\|^{2}}{\int_{M} V}$,

then $\Delta^{0}+V>0$.

Received by the editors February 15, 1988 and, in revised form, July 7, 1988.

1980 Mathematics Subject Classification (1985 Revision). Primary 53C20; Secondary 58G11, 58G32, 58C40.

The first author was partially supported by the SERC and the second author by the NSF. 
Here $\lambda_{1}$ is the first nonzero eigenvalue of $\Delta^{0}, V_{\min }$ is the minimum of $V$, $V_{a v}=\operatorname{vol}(M)^{-1} \int V$ and $\|\cdot\|$ is the $L^{2}$-norm. We set $V=\min \left\{R_{0}, \operatorname{Ric}^{\prime}\right\}$. Then for $\varepsilon^{\prime}$ and $\delta$ sufficiently small, (i) holds and the right side of (ii) is arbitrarily close to zero. However, by Myers' Theorem, the diameter of $M-A$ and hence of $M$ is bounded above. By Gromov [5] or Li and Yau [8], this keeps $\lambda_{1}$ bounded away from zero as $\varepsilon^{\prime}, \delta$ go to zero. Thus $\Delta^{0}+V>0$ and hence $\Delta^{0}+\mathrm{Ric}^{\prime}>0$.

To derive Theorem 1, we strengthen Lemma 2. If $\Delta^{0} f=\lambda_{1} f$, then we apparently need $\lambda_{1} \geq-V_{\min }$ to show $\left\langle\left(\Delta^{0}+V\right) f, f\right\rangle>0$. However, we can do much better provided $f$ is not concentrated near $V_{\min }$. In fact, by estimates of $\mathrm{Li}$ [7] and Croke [2] we can estimate how concentrated any function in the span of the first $m$ eigenfunctions of $\Delta^{0}$ may be near $V_{\min }$. Roughly speaking, this allows $V_{\min }=\mathrm{Ric}_{\min }^{\prime}$ to be arbitrarily negative and to replace $\lambda_{1}$ by $\lambda_{m}$ in Lemma 2(ii). Now we can mimic the proof of Theorem "' using the estimates in Li and Yau [9] for $\lambda_{m}$. The method of proof yields explicit upper bounds for $\varepsilon, \varepsilon^{\prime}$, and $|\delta|$ in terms of the geometric data.

A different method of coupling geometric information with semigroup domination may be found in [1].

3. Results for $\pi_{1}(M)$. Here the results for deep and shallow wells differ.

TheOREM 3. Assume $M$ admits a metric $g$ with $\operatorname{Ric}(x) \geq R_{0}>0$ except on a set $A$, with $\operatorname{diam}(A) \leq \varepsilon$, where $\operatorname{Ric}(x) \geq K$, for $\varepsilon$ as in Theorem 1 . If $\pi_{1}(M)$ contains a solvable subgroup of finite index, then $\pi_{1}(M)$ is finite. In particular, if $\pi_{1}(M)$ has polynomial growth, then $\pi_{1}(M)$ is finite [4].

As opposed to Myers' theorem, the proof uses $H^{1}=0$ to show $\pi_{1}$ is finite. In the tower of coverings $\tilde{M} \rightarrow M_{k} \rightarrow M_{k-1} \rightarrow \cdots \rightarrow M_{0} \rightarrow M$ associated to the solvable subgroup, we argue inductively that $H^{1}\left(M_{j} ; \mathbf{R}\right)=$ 0 implies $M_{j+1}$ is a finite cover of $M_{j}$, noting that $\Delta^{0}+\mathrm{Ric}^{\prime}$ is still positive for finite covers of $M$.

If a manifold with infinite $\pi_{1}$ admits a shallow well metric, the metric must be very distorted, in the sense that either the injectivity radius is very small at each point, or a generator of $\pi_{1}$ has very long geodesic length. To be more precise, we fix a point $x_{0}$ of $M$.

THEOREM $3^{\prime}$. Suppose $\pi_{1}\left(M, x_{0}\right)$ is infinite. For a set of generators $G=$ $\left\{\gamma_{1}, \ldots, \gamma_{t}\right\}$ for $\pi_{1}\left(M, x_{0}\right)$ and for positive numbers $l, \rho$ and $R_{0}$, there exist $\delta=\delta\left(R_{0}, \operatorname{dim} M, G, l, \rho, \pi_{1}\left(M, x_{0}\right)\right)<0$ and $\varepsilon=\varepsilon\left(R_{0}, \operatorname{dim} M\right)>0$ such that if $g$ is a metric satisfying

(i) some point of $M$ has injectivity radius larger than $\rho$,

(ii) the shortest geodesic in $\gamma_{i}$ has length less than $l$ for each $i$,

(iii) $\operatorname{Ric}(g) \geq R_{0}$ except on a set of diameter less than $\varepsilon$, then $\operatorname{Ric}(g)<\delta$ somewhere on $M$.

Here we bound the growth function $\gamma(r)$ of $\pi_{1}$ by $C_{1} \cdot \exp \left(C_{2} \sqrt{-\delta} r\right)$ for positive constants $C_{1}, C_{2}$ as in $[4,11]$. For fixed $C_{3}>0$ and $N \in \mathbf{Z}^{+}$, this is bounded in turn by $C_{3} \cdot r$ for $r=1,2, \ldots, N$ by taking $\delta$ close to zero. 
For $N$ sufficiently large, this implies $\pi_{1}(M)$ contains a nilpotent subgroup of finite index [4] and Theorem 3 applies.

4. Results for $p$-forms. $H^{p}(M, \mathbf{R})=0$ if $R^{p}$ is positive. More generally, if we define $R^{p^{\prime}}$ analogously to $\operatorname{Ric}^{\prime}$, then $H^{p}(M, \mathbf{R})=0$ whenever

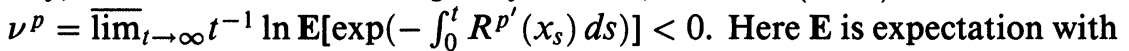
respect to the Wiener measure for Brownian motion $x_{s}$ on $M$. For the universal cover $\tilde{M}, \nu^{p}(M)=\nu^{p}(\tilde{M})$ with the pullback metric, so $\nu^{p}<0$ implies the vanishing of the space of $L^{2}$ harmonic $p$-forms on $\tilde{M}$. By the weak Hodge Theorem, $\operatorname{Im}\left[H_{c}^{p}(\tilde{M} ; \mathbf{R}) \rightarrow H^{p}(\tilde{M} ; \mathbf{R})\right]=0$, where $H_{c}^{p}$ denotes cohomology with compact supports. This implies that no nonzero class in $H^{p}(\tilde{M} ; \mathbf{R})$ has a representative differential form with compact support. For $p=1$, we showed in [3] that in fact $H_{c}^{1}(\tilde{M} ; \mathbf{Z})=0$, so in particular a compact 3-manifold with infinite $\pi_{1}$ and admitting a metric as in Theorem 3 must be a $K(\pi, 1)$.

For higher dimensional manifolds, we fix generators of $\pi_{1}(M)$ with associated growth function $\gamma(r)$ and a function $f(r)$ with $\limsup _{r \rightarrow \infty} f(r) \gamma(k r)$ $=0$ for all $k \in \mathbf{Z}^{+} \cdot f(r)$ is then independent of the choice of generators.

THEOREM 4. Assume $R^{p}>0$ or more generally that $\nu^{p}<0$ on $M$. Let $r$ denote the distance from a fixed point in $\tilde{M}$. If $\pi_{1}(M)$ is infinite, no nonzero class in $H^{p}(\tilde{M} ; \mathbf{R})$ has a representative form which decays faster than $f(r)$.

By Micallef-Moore [10], a simply connected manifold with curvature operator positive on complex totally isotropic two-planes is homeomorphic to a sphere $(\operatorname{dim} M \geq 4)$. It is known that this curvature condition implies $R^{2}>0$ if $\operatorname{dim} M$ is even, and it may be that it implies $R^{p}>0$ for $p \neq$ $1, n-1$. Thus Theorem 4 gives topological information on nonsimply connected manifolds with this type of curvature operator, at least for $p=2$ and $\operatorname{dim} M$ even.

To prove Theorem 4, we use a notion of bounded homology $H_{p}^{\infty}$ and $l_{1}$-cohomology $H_{1}^{p}$ complementary to Gromov's bounded cohomology [6]. As in [3, Theorem 5A], the integral of a compactly supported closed $p$ form over a bounded chain is unchanged under the heat flow and decays to zero as $t \rightarrow \infty$, so $\operatorname{Im}\left[H_{c}^{p} \rightarrow H_{1}^{p}\right]=0$. Using a Poincaré duality map in this theory and the fact that $\nu^{p}=\nu^{n-p}$, we conclude that every class $\alpha \in H_{p}(\tilde{M})$ is the boundary of an infinite chain $\sigma=\sum n_{i} \sigma_{i}$ with bounded coefficients. Let $\theta$ be a closed differential form which decays faster than $f(r)$. By estimates in [11], the boundary of suitable partial sums of $\sigma$ has volume growth bounded by $\gamma(k r)$ for some $k$, so the integral of $\theta$ over the boundary of these partial sums tends to zero at infinity. Thus $\int_{\alpha} \theta=0$ so $\theta$ is cohomologous to zero.

\section{REFERENCES}

1. P. Bérard and G. Besson, Number of bound states and estimates for some geometric invariants, Preprint.

2. C. Croke, Some isoperimetric inequalities and eigenvalue estimates, Ann. Sci. École Norm. Sup. Paris 13 (1980), 419-435. 
3. K. D. Elworthy and S. Rosenberg, Generalized Bochner Theorems and the spectrum of complete manifolds, Acta Math. Appl. (to appear).

4. M. Gromov, Groups of polynomial growth and expanding maps, Inst. Hautes Études Sci. Publ. Math. 53 (1981), 53-78.

5. __, Paul Levy's isoperimetric constant, Inst. Hautes Études Sci. Publ. Math., preprint, 1980.

6. __ Volume and bounded cohomology, Inst. Hautes Études Sci. Publ. Math. 56 (1982), 5-100.

7. P. Li, On the Sobolev constant and the p-spectrum of a compact Riemannian manifold, Ann. Sci. École Norm. Sup. Paris 13 (1980), 451-469.

8. P. Li and S. T. Yau, Estimates of eigenvalues on a compact Riemannian manifold, Proc. Sympos. Pure Math., vol. 36, Amer. Math. Soc., Providence, R.I., 1980, pp. 205-240.

9. __ On the parabolic kernel of the Schrödinger operator, Acta Math. 156 (1986), 153201.

10. M. Micallef and J. D. Moore, Minimal two-spheres and the topology of manifolds with positive curvature on totally isotropic two-planes, Ann. of Math. (2) 127 (1988), 199-227.

11. J. Milnor, A note on curvature and fundamental group, J. Differential Geom. 2 (1968), $1-7$.

Mathematics Institute, University of Warwick, Coventry CV4 7AL, United KINGDOM

Department of Mathematics, Boston University, Boston, Massachusetts 02215 